\title{
RELATIVE SOLIDITY FOR SPACES OF HOLOMORPHIC FUNCTIONS
}

\author{
By Stephen M. BuCKLEY \\ Department of Mathematics, NUI Maynooth
}

\begin{abstract}
We define and investigate relative solidity for sequence spaces, and use it to study spaces of holomorphic functions on the unit disk and related coefficient multiplier spaces. In particular, we find the multipliers $(l(u, v), H(p, q, t))$ and $(H(p, q, t), l(u, v))$ for many values of the parameters $u, v, p, q, t$.
\end{abstract}

\section{Introduction}

In this paper, we study sequence spaces associated with holomorphic functions on the unit disk $\mathbb{D} \subset \mathbb{C}$, identifying any such function $f$ with its Taylor sequence $(\hat{f}(n))$. The concept of solidity, which goes back to the 1960's (see [9], [16]) is useful in the often difficult task of determining whether or not a given sequence lies in a given function space (such as a particular Hardy or Bergman space) or space of multipliers involving such spaces. We introduce the more general notion of relative solidity, examine it in detail for the function spaces $H(p, q, t)$, and thereby characterize in most cases the multipliers from $H(p, q, t)$ to $l(u, v)$ (and also in the opposite direction). This one method of characterizing these multiplier spaces replaces a variety of other methods that have been used in the literature to characterize these multipliers in certain cases, notably by Blasco [3] and Jevtić and Pavlović [11]. In the latter paper, multipliers from $H(p, q, t)$ to $l^{u}=l(u, u)$ were classified in most cases, and the answer was conjectured in the missing case. We disprove this conjecture in Section 5 and, although we cannot completely classify the multipliers in this case, we find separate necessary and sufficient conditions which suggest that any answer must be rather complicated; see Remarks 3 and 4 in Section 5.

Recall that a sequence space $X$ is solid if $\left(b_{n}\right) \in X$ whenever $\left(a_{n}\right) \in X$ and $\left|b_{n}\right| \leq\left|a_{n}\right|$. More generally, we define $S(X)$ and $s(X)$, the solid hull and solid core of $X$, respectively, by the equations

$$
\begin{aligned}
& S(X)=\left\{\left(\lambda_{n}\right): \exists\left(a_{n}\right) \in X \text { such that }\left|\lambda_{n}\right| \leq\left|a_{n}\right|\right\}, \\
& s(X)=\left\{\left(\lambda_{n}\right):\left(\lambda_{n} a_{n}\right) \in X \text { whenever }\left(a_{n}\right) \in l^{\infty}\right\} .
\end{aligned}
$$

For more on solidity, see [1].

1991 Mathematics Subject Classification. 30H05, 42A45.

The author was supported in part by Forbairt. 
It is easy to determine $S(X)$ or $s(X)$ for some non-solid spaces $X$, but not for others. For example, $S\left(H^{p}\right)=s\left(H^{q}\right)=H^{2}$ for $q \leq 2 \leq p$, but these spaces are unknown if $p<2<q$ (the positive results follow from the work of Littlewood on random series [13]; see also [7, Theorem A.5]). We define the more general concepts $S_{F}(X)$ and $s_{F}(X)$, the hull and core of $X$ relative to $F, F$ being a family of spaces. We can gain new information about $X$ in cases where $S(X)$ or $s(X)$ are unknown by making a judicious choice of $F$; in particular $F$ should be such that $S_{F}(X)$ and $s_{F}(X)$ can be determined.

To give a concrete example, we need some notation. For $t \in \mathbb{R}$, we write $D^{t}$ for the sequence $\left((n+1)^{t}\right)$, for all $n \geq 0$. If $\lambda=\left(\lambda_{n}\right)$ is a sequence and $X$ a sequence space, we write $\lambda X=\left\{\left(\lambda_{n} x_{n}\right):\left(x_{n}\right) \in X\right\}$; thus, for example, $\left(a_{n}\right) \in D^{t} l^{\infty}$ if and only if $\left|a_{n}\right|=O\left(n^{t}\right)$. If $X, Y$ are two sequence spaces, we write $X Y=\bigcup_{x \in X} x Y$ (XY thus consists of all convolutions of $X$ and $Y$ functions, if $X$ and $Y$ can be viewed as function spaces).

For $1<p<2$, Bergman space $A^{p}$ is known to be a subset of $A \equiv D^{1 / p} l^{\infty}, B \equiv$ $D^{3 / p-1} l^{p}$, and $C \equiv D^{1 / p} l^{p /(p-1)}$ (see [15] for the first two, and [10] for the third), but how are these containments related? Clearly $C$ is a proper subset of $A$ and, after a little calculation, we see that neither $B$ nor $C$ is a subset of the other. Thus a sharper containment is given by $A^{p} \subset X \equiv B \cap C$. Letting $F_{1}$ be the family of spaces of the form $D^{t} l^{r}, t \in \mathbb{R}, r>0$, it is natural to ask if an even sharper containment is possible by intersecting $X$ with some other $Y \in F_{1}$. We shall see that this is not the case, so that $X$ is the intersection of all such containing spaces, a fact that we denote by $X=S_{1}\left(A^{p}\right)$. Both $S\left(A^{p}\right)$ and $S_{1}\left(A^{p}\right)$ are intersections of all containing spaces in some family (all solid spaces or $\left.F_{1}\right)$ and, since $F_{1}$ spaces are all solid, $S\left(A^{p}\right) \subset S_{1}\left(A^{p}\right)$. We shall in fact determine the hull and core of $A^{p}$ relative to $F_{1}$ for all values of $p$, but we shall also see that the family $F_{2}$ of all mixed-norm spaces $D^{t} l(r, s)$ gives simpler and more useful relative hulls and cores than does $F_{1}$, and it is these that we use to classify multiplier spaces.

After the preliminaries of Section 1, we give some preparatory mixed-norm results in Section 2, and discuss relative hulls and cores in Section 3. In Section 4, we completely classify $S_{i} H(p, q, t)$ and $s_{i} H(p, q, t), i=1,2$, and in Section 5, we examine multipliers between $l(u, v)$ and $H(p, q, t)$.

The author wishes to thank M.S. Ramanujan and D. Vukotić for helpful discussions related to this paper.

\section{Notation and terminology}

As mentioned already, $\mathbb{D}=\{|z|<1\}$ is the unit disk in the complex plane, $d A(z)=$ $\pi^{-1} d x d y(z=x+i y)$ is the normalized Lebesgue area measure on $\mathbb{D}, \mathcal{H}(\mathbb{D})$ is the algebra of holomorphic functions in $\mathbb{D}$. For $f \in \mathcal{H}(\mathbb{D}), 0<r<1,0<p \leq \infty$, the integral means $M_{p}(r, f)$ are defined by $M_{p}(r, f)=\left(\frac{1}{2 \pi} \int_{0}^{2 \pi}\left|f\left(r e^{i \theta}\right)\right|^{p} d \theta\right)^{1 / p}$, with the usual modification when $p=\infty$. The Hardy space $H^{p}, 0<p \leq \infty$, consists of all $f \in \mathcal{H}(\mathbb{D})$ for which $\|f\|_{H^{p}}=\lim _{r \rightarrow 1} M_{p}(r, f)<\infty$, and $H(p, q, t), 0<p \leq \infty, 0<q, t<\infty$, consists of all $f \in \mathcal{H}(\mathbb{D})$ for which

$$
\|f\|_{H(p, q, t)} \equiv\left(\int_{0}^{1} M_{p}(r, f)^{q}(1-r)^{t q-1} d r\right)^{1 / q}<\infty .
$$


We also define $H(p, \infty, t)$ (often denoted $\left.H_{t}^{p}\right)$ to consist of all $f \in \mathcal{H}(\mathbb{D})$ for which

$$
\|f\|_{H(p, \infty, t)} \equiv \sup _{0<r<1}(1-r)^{t} M_{p}(r, f)<\infty .
$$

When we define fractional differentiation below, we shall extend the definition of $H(p, q, t)$ to all $t \in \mathbb{R}$ (and all $0<p, q \leq \infty$ ). The Bergman space $A^{p}, 0<p<\infty$, is defined to be $H(p, p, 1 / p)$; equivalently, $f \in A^{p}$ if $\|f\|_{A^{p}}^{p}=\int_{\mathbb{D}}|f(z)|^{p} d A(z)<\infty$.

A sequence space means a vector subspace of $\mathbb{C}^{\mathbb{N}}$. Whenever $\lambda$ is used to denote a sequence, $\lambda_{n}$ denotes its $n$th term. A sequence $\lambda$ is a multiplier from one sequence space $X$ to another one $Y$, denoted $\lambda \in(X, Y)$, if $\left(\lambda_{n} a_{n}\right) \in Y$ whenever $a \in X$.

The sequence $D^{s}=\left((n+1)^{s}\right)$ already defined is a particularly important multiplier between many pairs of function spaces; the associated operator, also denoted $D^{s}$ will be referred to as fractional differentiation of order $s$. It follows from the work of Flett [8] that $D^{s} H(p, q, t)=H(p, q, s+t)$, for all $s, t>0$. One can thus consistently extend the definition of $H(p, q, t)$ to the case $t \leq 0$ by defining $H(p, q, t)=D^{-s} H(p, q, s+t)$ for any $s>-t$; in particular, $H(\infty, \infty, 0)$ is the well-known space $\mathcal{B}$ of Bloch functions. If $X$ has a norm, then we define a norm on $D^{s} X$ by pullback (the same goes for quasinorms, or other weaker versions of norms). Let us agree to define $\|f\|_{H(p, q, t)}=\left\|D^{1-t} f\right\|_{H(p, q, 1)}$ for all $t \leq 0$. Our definition of $H(p, \infty, t)$ for $t>0$ might lead one to expect that $H(p, \infty, 0)=H^{p}$, but this is not the case. For example, $f(z)=1 /(1-z)$ belongs to $H(1, \infty, 0)$ (since $f^{\prime} \in H(1, \infty, 1)$ ), but $f \notin H^{1}$.

We adopt the conventions $1 / 0=\infty, 0 \cdot \infty=0, y / \infty=0$, and $x^{0}=1$, whenever $x, y \geq 0$. For any exponent $1<p<\infty, p^{\prime}$ always denotes the dual exponent $p /(p-1)$; also $1^{\prime}=\infty$ and $\infty^{\prime}=1$. In proofs, we shall use $C$ to denote any harmless constant that does not affect the argument; it can change from one instance to the next. If $A$ and $B$ are positive quantities, $A \lesssim B$ means that $A \leq C B$ for some such $C$, and $A \approx B$ means that $A \lesssim B$ and $B \lesssim A$.

\section{2. "Mixed-norm" spaces}

Let us pause to gather some results on mixed norms which will later be useful. It is sometimes convenient to write sequences $\left(a_{n}\right)_{n=0}^{\infty}$ as formal power series $\sum_{n=0}^{\infty} a_{n} x^{n}$. These series will always involve powers of $x$ to distinguish them from analytic functions which are written as power series in $z$; in such cases, we care only about the sequence involved, and not about the function values or convergence. We define $I_{k}$ to be the $k t h$ dyadic block of integers, i.e. $I_{k}=\left\{2^{k-1}, \ldots, 2^{k}-1\right\}$ for $k>0$, and $I_{0}=\{0\}$. On any sequence space, we define the operator $S_{k}, k \geq 0$, which selects out the $k$ th dyadic block of terms and shifts it to an initial position. More precisely, $S_{0}\left(\sum_{n=0}^{\infty} a_{n} x^{n}\right)=a_{0} x^{0}$ and $S_{k}\left(\sum_{n=0}^{\infty} a_{n} x^{n}\right)=\sum_{n \in I_{k}} a_{n} x^{n-2^{k-1}}$. We also define the multiplier $\Delta_{k}=\sum_{n \in I_{k}} x^{n}$; note that $S_{k}$ is simply a shift operator composed with $\Delta_{k}$.

Suppose $A, B$ are (quasi-)normed sequence spaces. Essentially as in [6], the (quasi)normed space $A[B]$ is defined as the set of all sequences $\lambda$ such that

$$
\|\lambda\|_{A[B]}=\left\|\left(\left\|S_{k} \lambda\right\|_{B}\right)_{k=0}^{\infty}\right\|_{A}<\infty .
$$


We can iterate this construction to define $\|\lambda\|_{A[B[C]]}=\left\|\left(\left\|S_{k} \lambda\right\|_{B[C]}\right)_{k=0}^{\infty}\right\|_{A}$, and so on. This bracketing operation is non-associative, so it is important to note that all our iterated constructions are constructed "from the inside outwards" as here. $D^{t}$ is always "applied last", so that $D^{t} A[B]$ refers to the space of sequences $D^{t} c$, where $c \in A[B]$, and never to the mixed norm space $C[B], C=D^{t} A$.

Taking $A$ and $B$ to be spaces of the form $l^{r}, 0<r \leq \infty$ (with the usual quasinorm attached), we get the spaces $l^{q}\left[l^{p}\right]$. We often use the more common notation $l(p, q) \equiv$ $l^{q}\left[l^{p}\right]$ and $\|\cdot\|_{p, q} \equiv\|\cdot\|_{l^{q}\left[l^{p}\right]}$; similarly, $l(p, q, r) \equiv l^{r}\left[l^{q}\left[l^{p}\right]\right]$, and so on. Observe that $l^{p}=l(p, p)=l(p, p, p)$, etc. We mainly use the mixed-norm construction in the form $X \equiv X_{1}\left[\ldots\left[X_{n}\right] \ldots\right]$, where $X_{i} \in F \equiv\left\{l^{p}: 0<p \leq \infty\right\}, 1 \leq i<n$, and $X_{n} \in F \cup\left\{H^{p}\right.$ : $1<p<\infty\}$.

Let $g:[0, \infty) \rightarrow \mathbb{R}$ be an infinitely differentiable non-increasing function which equals 1 on $[0,1]$, and 0 on $[2, \infty)$. Let $\phi(t)=g(t / 2)-g(t)$, and define $w_{k, n}=\phi\left(n 2^{1-k}\right)$, for $n \geq 0, k>0, w_{0,0}=w_{0,1}=1$, and $w_{0, n}=0$ if $n>1$. We now define the sequence $\widetilde{\Delta}_{k}=$ $\left(w_{k, n}\right)_{n=0}^{\infty}$; this is a "smoothened" version of $\Delta_{k}$. Note that $0 \leq w_{k, n} \leq 1, \sum_{k=0}^{\infty} w_{k, n}=1$, and $w_{n, k}$ is zero if $n$ lies outside $I_{k} \cup I_{k+1}$.

Lemma 2.1. Let $t \in \mathbb{R}$. Then

$$
\begin{aligned}
& \|f\|_{H(p, q, t)} \approx\left\|\left(2^{-k t}\left\|\Delta_{k} f\right\|_{H^{p}}\right)\right\|_{l^{q}}, \quad 1<p<\infty, 0<q \leq \infty . \\
& \|f\|_{H(\infty, q, t)} \lesssim\left\|\left(2^{-k t}\left\|\Delta_{k} f\right\|_{H^{\infty}}\right)\right\|_{l^{q}}, \quad 0<q \leq \infty . \\
& H(p, q, t)=D^{t} l^{q}\left[H^{p}\right], \quad 1<p<\infty, 0<q \leq \infty . \\
& H(\infty, q, t) \supset D^{t} l^{q}\left[H^{\infty}\right], \quad 0<q \leq \infty . \\
& \|f\|_{H(p, q, t)} \approx\left\|\left(2^{-k t}\left\|\widetilde{\Delta}_{k} f\right\|_{H^{p}}\right)\right\|_{l^{q}}, \quad 0<p \leq 1,0<q<\infty .
\end{aligned}
$$

Proof. (2.1) and (2.1') are due to Mateljević and Pavlović [14], (2.2) was proved in [4], and $(2.3)$ is due to Blasco [3], so it remains to prove $\left(2.2^{\prime}\right)$.

We assume, as we may, that $t>0$. Let $f_{k}=\Delta_{k} f$. By $\left(2.1^{\prime}\right)$, we see that it suffices to show that $2^{-k t}\left\|f_{k}\right\|_{H^{\infty}}$ and $\left\|D^{-t} f_{k}\right\|_{H^{\infty}}$ are comparable for $k>0$ (with constants of comparability depending only on $t>0$ ). To see this, we first claim that $2^{-k s}\left\|f_{k}\right\|_{H^{\infty}} \approx$ $\left\|f_{k}\right\|_{H(\infty, q, s)}$. Since $f_{k}$ has zero coefficients beyond position $2^{k}$, we have $r^{2^{k}}\left\|f_{k}\right\|_{H^{\infty}} \leq$ $M_{\infty}(r, f)$ (see [14, Lemma 3.1]). Taking $1-r \in\left[2^{-k}, 2^{-k-1}\right]$, we see that $r^{2^{k}} \approx 1$. Routine estimation now gives $2^{-k s}\left\|f_{k}\right\|_{H^{\infty}} \lesssim\left\|f_{k}\right\|_{H(\infty, q, s)}$. The opposite direction of our claim follows from $\left(2 \cdot 1^{\prime}\right)$.

Using our claim twice, and the fact that $H(\infty, q, t+1)=D^{t} H(\infty, q, 1)$, we get

$$
2^{-k t}\left\|f_{k}\right\|_{H^{\infty}} \approx 2^{k}\left\|f_{k}\right\|_{H(\infty, q, t+1)} \approx 2^{k}\left\|D^{-t} f_{k}\right\|_{H(\infty, q, 1)} \approx\left\|D^{-t} f_{k}\right\|_{H^{p}} .
$$

By a lacunary sequence, we mean any sequence which has at most one non-zero term in every dyadic block (we shall only have need for sequences whose non-zero terms occur only at positions which are a power of 2). Lemma 2.1 has as corollaries the following pair of lemmas characterizing the lacunary and monotonic sequences that lie in $H(p, q, s)$. The first lemma is a trivial corollary, while the second one follows from Corollary 1.3 of [4]. 
Lemma 2.2. For any $t \in \mathbb{R}, 0<p<\infty, 0<q \leq \infty$, a lacunary sequence lies in $H(p, q, t)$ if and only if it lies in $D^{t} l^{q}$.

Lemma 2.3. Suppose that $a_{n} \geq 0$ for all $n \geq 0$, and that $t \in \mathbb{R}, 1<p<\infty, 0<$ $C_{1}<\infty, 0<q \leq \infty$. If $\left(a_{n}\right)$ is monotonic then $\left(a_{n}\right) \in H(p, q, t)$ if and only if $\left(a_{n}\right) \in$ $D^{t+1 / p+1 / q-1} l^{q}$. Furthermore, the norms of $\left(a_{n}\right)$ in these two spaces are comparable, with constants of comparability depending only on $p, q, t$.

We now state a theorem in [4] which we shall need in Section 5; parts of it can also be found in [12] and [17].

Lemma 2.4. The following containments hold, and all are proper if $p \neq 2$.

$$
\begin{array}{llllll}
l\left(p^{\prime}, 2, \ldots, 2\right) & \supset l^{2}\left[l^{2}\left[\cdots l^{2}\left[H^{p}\right] \cdots\right]\right] \supset H^{p} \supset l^{p}\left[l^{p}\left[\cdots l^{p}\left[H^{p}\right] \cdots\right]\right], & & 1<p \leq 2, \\
l\left(p^{\prime}, 2, \ldots, 2\right) & \subset l^{2}\left[l^{2}\left[\cdots l^{2}\left[H^{p}\right] \cdots\right]\right] \subset H^{p} \subset l^{p}\left[l^{p}\left[\cdots l^{p}\left[H^{p}\right] \cdots\right]\right], & & 2 \leq p<\infty .
\end{array}
$$

We next characterize $S(H(p, q, t))$ and $s(H(p, q, t))$ when this is easy, and give partial results in other cases; this lemma gives the easy half of Theorem 4.2 in all cases, and suggests why the spaces $D^{t} l(u, v)$ are naturally associated with $H(p, q, t)$.

Lemma 2.5. Let $0<q \leq \infty$, and $t \in \mathbb{R}$. Then

$$
\begin{aligned}
& S(H(p, q, t))=H(2, q, t)=D^{t} l(2, q), \quad 2 \leq p \leq \infty, \\
& s(H(p, q, t))=H(2, q, t)=D^{t} l(2, q), \quad 0<p \leq 2, \\
& H(p, q, t) \subset \begin{cases}D^{t} l\left(p^{\prime}, q\right), & 1<p \leq 2, \\
D^{t+1 / p-1} l(\infty, q), & 0<p \leq 1,\end{cases} \\
& H(p, q, t) \supset D^{t} l\left(p^{\prime}, q\right), \quad 2 \leq p \leq \infty \text {. }
\end{aligned}
$$

Proof. (2.4) is proved in [2, Theorem 1.8] and [14, Theorem 2.5]. For (2.5), we refer the reader to [4, Lemma 1.2]. Corollaries 3.1 and 3.2 of [3] imply the case $q<\infty$ of (2.6), and the case $p<\infty, 1<q<\infty$ of (2.7). We prove both containments in all cases.

Combining (2.2) and the Hausdorff-Young Theorem gives (2.6) for $1<p \leq 2$, and (2.7) for $p<\infty$; similarly, the case $p=\infty$ of (2.7) follows by using (2.2') in place of (2.2). To get (2.6) for $p \leq 1$, we instead combine (2.3) and the containment $H^{p} \subset D^{1 / p-1} l^{\infty}$ (for which, see [7, Theorem 6.4$]$ ); it is now easy to replace $\widetilde{\Delta}_{k}$ in the resulting expression by $\Delta_{k}$ to get the desired containment.

Lemma 2.6. Let $t \in \mathbb{R}, 0<p, q \leq \infty$, and let $s_{0}=\max \{t, t+1 / p-1\}$. Then $D^{s} l^{\infty}$ contains $H(p, q, t)$ for all $s \geq s_{0}$, but not for any $s<s_{0}$.

Proof. We may assume $t>0$ without loss of generality. Containment for $s=s_{0}$ follows from Lemma 2.5. To see that containment is false for $s<t$, we use Lemma 2.2 to get that $\sum_{n=1}^{\infty} n^{s+\epsilon} z^{2^{n}}$ lies in $H(p, q, t) \backslash D^{s} l^{\infty}$ whenever $0<\epsilon<t-s$.

As the reader may verify, $f_{t}(z) \equiv(1-z)^{-s}$ lies in $H(p, q, t)$ for all $s<t+1 / p$ (and for no other $s$, except $s=t+1 / p$, if $q=\infty)$; as a hint, the first step is to verify the estimate 
$\int_{0}^{2 \pi}\left|1-r e^{i \theta}\right|^{-1-\epsilon} d \theta=O\left((1-r)^{-\epsilon}\right), \epsilon>0$. Moreover, the $n$th coefficient of $f_{t}$ is asymptotic to $\Gamma(s)^{-1} n^{s-1}$. Consequently, $D^{s} l^{\infty}$ does not contain $H(p, q, t)$ if $s<t+1 / p-1$.

Our next lemma characterizes when fractional differentiation takes one $l(p, q)$ space to another; we sketch the proof for completeness. We shall not need a similar lemma for spaces $l\left(p_{1}, \ldots, p_{k}\right)$ for $k>2$, but let us point out two easily verified facts. First, $l\left(p_{1}, \ldots, p_{k}\right) \subset l\left(q_{1}, \ldots, q_{k}\right)$ if $p_{i} \leq q_{i}, i=1, \ldots, k$, and secondly the spaces $l\left(p_{1}, \ldots, p_{m}\right)$ and $l\left(q_{1}, \ldots, q_{k}\right), k<m$, are equal if and only if $p_{1}=p_{j}$ for all $j<m-k+1$ and $p_{m-i}=q_{k-i}, i=0, \ldots, k-1$.

Lemma 2.7. Given $0<a, b, c, d \leq \infty$, we have $l(a, b) \subset D^{t} l(c, d)$ if and only if:

(i) $t \geq 0$, if $a \leq c$ and $b \leq d$;

(ii) $t>0$, if $a \leq c$ and $b>d$;

(iii) $t \geq 1 / c-1 / a$, if $a>c$ and $b \leq d$;

(iv) $t>1 / c-1 / a$, if $a>c$ and $b>d$.

Sketch of Proof. Using the fact that $p \mapsto\|\cdot\|_{l^{p}}$ is decreasing and/or Hölder's inequality, containment follows readily in each case. It remains to prove sharpness of the lower bound on $t$. For (i), note that the sequence $\sum_{n=2}^{\infty} n^{-1 / a}(\log n)^{-2 / b} x^{2^{n}}$ belongs to $l(a, b) \backslash D^{t} l^{\infty}$, for all $t<0$. For (ii), the lacunary sequence $\sum_{n=1}^{\infty} n^{-1 / d} x^{2^{n}}$ lies in $l(a, b) \backslash l(\infty, d)$. For (iii), it suffices to note that $\left(n^{-1 / a-\epsilon / 2}\right)_{1}^{\infty} \in l(a, b) \backslash D^{t} l(c, \infty)$ whenever $t=1 / c-1 / a-\epsilon, \epsilon>0$. For (iv), we have $\left(n^{-1 / a}(\log n)^{-1 / d}\right)_{2}^{\infty} \in l(a, b) \backslash D^{1 / c-1 / a} l(c, d)$.

For all $0<p, q \leq \infty$, we define the binary operations $p \ominus q=r$ and $p \oplus q=s$, where $1 / r=\max \{1 / p-1 / q, 0\}, 1 / s=1 / q+1 / p$. The next lemma is essentially due to Kellogg $[12]$.

Lemma 2.8. If $0<a, b, c, d \leq \infty$, then $(l(a, b), l(c, d))=l(c \ominus a, d \ominus b)$.

If $Y, W$ are quasi-normed spaces, we associate with $(Y, W)$ and $Y W$ the quasi-norms

$$
\begin{aligned}
\|\lambda\|_{(Y, W)} & =\sup \left\{\left\|\left(\lambda_{n} y_{n}\right)\right\|_{W}:\|y\|_{Y} \leq 1\right\}, \\
\|x\|_{Y W} & =\inf \left\{\|y\|_{Y}\|w\|_{W}:\left(x_{n}\right)=\left(y_{n} w_{n}\right), y \in Y, w \in W\right\},
\end{aligned}
$$

We can thus define the spaces $(X, Z)[(Y, W)]$ and $X Z[Y W]$, whenever $X, Y, Z, W$ are quasinormed. The following lemma is a special case of [4, Theorem 2.10].

Lemma 2.9. Let $l^{*}=\left\{l^{p}: 0<p \leq \infty\right\}$ and $H^{*}=\left\{H^{p}: 1<p<\infty\right\}$. Suppose that $X_{i}, Y_{i} \in l^{*}$ for all $1 \leq i<n$, and that $X_{n}, Y_{n} \in H^{*} \cup l^{*}$. Then, writing $Z_{i}=\left(X_{i}, Y_{i}\right)$, $W_{i}=X_{i} Y_{i}$, we have:

$$
\begin{aligned}
\left(X_{1}\left[\ldots\left[X_{n}\right] \ldots\right], X_{1}\left[\ldots\left[X_{n}\right] \ldots\right]\right) & =Z_{1}\left[\ldots\left[Z_{n}\right] \ldots\right] \\
X_{1}\left[\ldots\left[X_{n}\right] \ldots\right] \cdot X_{1}\left[\ldots\left[X_{n}\right] \ldots\right] & =W_{1}\left[\ldots\left[W_{n}\right] \ldots\right],
\end{aligned}
$$

We shall use this lemma with $X_{n}=H^{p}$ in Section 5 but let us record here a couple of other easy consequences that we shall need later: if $0<p_{i}, q_{i} \leq \infty, 1 \leq i \leq n$, then

$$
\begin{aligned}
\left(l\left(p_{1}, \ldots, p_{n}\right), l\left(q_{1}, \ldots, q_{n}\right)\right) & =l\left(q_{1} \ominus p_{1}, \ldots, q_{n} \ominus p_{n}\right), \\
l\left(p_{1}, \ldots, p_{n}\right) \cdot l\left(q_{1}, \ldots, q_{n}\right) & =l\left(q_{1} \oplus p_{1}, \ldots, q_{n} \oplus p_{n}\right) .
\end{aligned}
$$


Note that (2.8), which generalizes Lemma 2.8, follows by using Lemma 2.8 to determine the spaces $Z_{i}$, while for $(2.9)$ we use the equation $l^{p} l^{q}=l^{q \oplus p}$, an easy application of Hölder's inequality.

\section{Relative hulls and cores}

Suppose $X$ is a sequence space. For a family $F$ of sequence spaces, the $F$-hull $S_{F}(X)$ of $X$ is the intersection of all spaces in $F$ that contain $X$, and the $F$-core $s_{F}(X)$ is the vector space sum of all spaces in $F$ contained in $X$. Note that if $\mathrm{F}$ is any family of solid spaces, then

$$
s_{F}(X) \subset s(X) \subset X \subset S(X) \subset S_{F}(X) .
$$

and $S_{F}(X)=S(X), s_{F}(X)=s(X)$, if $F$ is the class of all solid spaces.

$S_{F}(X)$ and $s_{F}(X)$ might not even be solid unless $F$ is a family of solid spaces. But, since we want to use simple auxiliary spaces to gain information about our original space, we shall consider only families of solid spaces. In fact, we shall mainly be interested in the families $F_{1}$ and $F_{2}$ (and especially $F_{2}$ ), where

$$
F_{k}=\left\{D^{t} l\left(p_{1}, \ldots, p_{k}\right): t \in \mathbb{R}, 0<p_{1}, \ldots, p_{k} \leq \infty\right\}, \quad k \in \mathbb{N} .
$$

For brevity, we shall write $s_{k}(X)=s_{F_{k}}(X), S_{k}(X)=S_{F_{k}}(X)$, for $k \in \mathbb{N}$.

As a warning to the reader, we note that unlike the ordinary notion of solidity, it is possible for $X$ to equal one of $S_{F}(X), s_{F}(X)$ without equalling the other. For instance, let $X=D^{1} l^{1} \cap l^{\infty}, Y=D^{1} l^{1}+l^{\infty}$, it is clear that $S_{F_{1}} X=X$ and $s_{F_{1}} Y=Y$, but it follows from Lemma 2.7 that $s_{F_{1}} X=\bigcup_{p<\infty} l^{p}$ and $S_{F_{1}} Y=\bigcap_{p>1} D^{1} l^{p}$, so that $\left(\log ^{-2}(n+2)\right)$ lies in $X \backslash\left(s_{F_{1}} X\right)$, while $(\log (n+2))$ lies in $\left(S_{F_{1}} Y\right) \backslash Y$.

We begin with a lemma classifying $s_{1}(X)$ and $S_{1}(X)$ when $X \in F_{2}$; note that $s_{1}(X), S_{1}(X) \in F_{1}$ only when $p=q$. With the help of Lemma 2.7, the proof is a straightforward case study which we leave to the reader.

Lemma 3.1. Suppose that $X \equiv D^{t} l(p, q) \in F_{2}$, and let $r=1 / q-1 / p$. Then

$$
\begin{array}{r}
s_{1}(X)= \begin{cases}D^{t}\left[l^{p}+D^{r} l^{q}\right], & p \leq q, \\
D^{t}\left[l^{q}+\bigcup_{\epsilon>0} D^{-\epsilon} l^{p}\right], & p>q,\end{cases} \\
S_{1}(X)= \begin{cases}D^{t}\left[l^{q} \cap\left(\bigcap_{\epsilon>0} D^{\epsilon} l^{p}\right)\right], & p<q, \\
D^{t}\left[l^{p} \cap D^{r} l^{q}\right], & p \geq q,\end{cases}
\end{array}
$$

Multiplier spaces have the following elementary properties.

(M1) If $X \subset(Y, Z)$ then $Y \subset(X, Z)$.

(M2) $\left(D^{t} X, Y\right)=D^{-t}(X, Y)$ and $\left(X, D^{t} Y\right)=D^{t}(X, Y)$.

(M3) If $X \subset Y$ then $(Z, X) \subset(Z, Y)$ and $(Y, Z) \subset(X, Z)$.

We end this section with a lemma which we apply with for $F=F_{k}, U \in F_{k}$ (in which case the closure hypotheses in the lemma hold, by (2.8), (2.9), and (M2)). Containment in parts (iii), (iv) of this lemma cannot be changed to equality; see the final paragraph in Section 5. 
Lemma 3.2. Suppose that $U, X$ are sequence spaces and that $F$ is a family of sequence spaces. Suppose also that $(A, U),(U, A), A U \in F$ for all $A \in F$. Then

(i) $s_{F}(X, U)=s_{F}\left(S_{F}(X), U\right)$;

(ii) $s_{F}(U, X)=s_{F}\left(U, s_{F}(X)\right)$;

(iii) $S_{F}(X, U) \subset S_{F}\left(s_{F}(X), U\right)$;

(iv) $S_{F}(U, X) \subset S_{F}\left(U, S_{F}(X)\right)$.

Moreover, if $S_{F}(X), s_{F}(X) \in F$, then

(i') $s_{F}(X, U)=\left(S_{F}(X), U\right)$;

(ii') $s_{F}(U, X)=\left(U, s_{F}(X)\right)$;

(iii') $S_{F}(X, U) \subset\left(s_{F}(X), U\right)$;

$\left(\mathrm{iv}^{\prime}\right) S_{F}(U, X) \subset\left(U, S_{F}(X)\right)$.

Proof. We first prove (i). Let $A \in F, A \subset(X, U)$. Then $X \subset(A, U) \in F$ and so $S_{F}(X) \subset(A, U)$. Thus $A \subset\left(S_{F}(X), U\right)$ and so $A \subset s_{F}\left(S_{F}(X), U\right)$. Since $A \subset(X, U)$ is arbitrary, $s_{F}(X, U) \subset s_{F}\left(S_{F}(X), U\right)$. The opposite containment is immediate by (M3).

The proof of (ii) is similar. If $A \in F, A \subset(U, X)$, then $X \supset A U \in F$ and so $A U \subset s_{F}(X)$. Thus $A \subset\left(U, s_{F}(X)\right)$ and so $A \subset s_{F}\left(U, s_{F}(X)\right)$. Since $A \subset(U, X)$ is arbitrary, $s_{F}(U, X) \subset s_{F}\left(U, s_{F}(X)\right)$. The opposite containment again follows by (M3).

Finally, (iii) and (iv) follow from (M3), and (i')-(iv') from the closure properties $(A, U),(U, A) \in F$.

\section{4. $S_{2} H(p, q, t)$ and $s_{2} H(p, q, t)$}

We shall need the following "Marcinkiewicz multiplier"-type theorem, which replaces Lemma 2.3 when $p \leq 1$.

Lemma 4.1. Suppose that $0<p \leq 1,0<q \leq \infty, t \in \mathbb{R}, u \in l^{q}$, and $1 / p<m \in \mathbb{N}$. Suppose also that $g:[1, \infty) \rightarrow \mathbb{R}$ is a $C^{m}$ function satisfying $\left|g^{(i)}(x)\right| \leq x^{t+1 / p-1-i} u_{k}$, for all $2^{k-1} \leq x<2^{k}, k \in \mathbb{N}$, and $0 \leq i \leq m$. Then $f(z) \equiv \sum_{n=1}^{\infty} g(n) z^{n} \in H(p, q, t)$, and $\|f\|_{H(p, q, t)} \leq C\|u\|_{l^{q}}$, for some $C=C(p, q, t)$.

Proof. We use Lemma 2.1 and the notation therein. We may assume that $m$ is the least integer greater than $1 / p$. Since the point-evaluation functionals are bounded in $H(p, q, t)$, we may also assume without loss of generality that $u_{k}=0$ for $k<2+\log _{2} m$. We assume $k \geq 2+\log _{2} m$ from now on. We first write

$$
\begin{aligned}
2 \pi\left\|\widetilde{\Delta}_{k} f\right\|_{H^{p}}^{p} & =M_{p}\left(1, \widetilde{\Delta}_{k} f\right)^{p} \\
& =\int_{|\theta| \leq 2^{-k}}\left|\widetilde{\Delta}_{k} f\left(e^{i \theta}\right)\right|^{p} d \theta+\int_{2^{-k}<|\theta| \leq \pi}\left|\widetilde{\Delta}_{k} f\left(e^{i \theta}\right)\right|^{p} d \theta \equiv I+I I .
\end{aligned}
$$

It suffices to show that $I, I I \lesssim 2^{k p t} v_{k}^{p}$, where $v_{k}=\sum_{j=k-1}^{k+2} u_{k}$, since (2.3) then gives

$$
\|f\|_{H(p, q, t)} \approx\left\|\left(2^{-k t}\left\|\widetilde{\Delta}_{k} f\right\|_{H^{p}}\right)\right\|_{l^{q}} \lesssim\left\|\left(v_{k}\right)\right\|_{l^{q}} \lesssim\left\|\left(u_{k}\right)\right\|_{l^{q}}, \quad q<\infty .
$$

The polynomial $\widetilde{\Delta}_{k} f$ has at most $2^{k+1}$ terms, and so the bound on $g^{(0)}$ alone gives $\left|\widetilde{\Delta}_{k} f\left(e^{i \theta}\right)\right|^{p} \lesssim 2^{(k+1) p} \cdot 2^{k(t p+1-p)}\left(u_{k}+u_{k+1}\right)^{p}$, for all $\theta \in \mathbb{R}$. Thus $I \lesssim 2^{k p t} v_{k}^{p}$. 
A repeated summation by parts argument will allow us to control $I I$. Let us write $\mathcal{D}^{j}$ for the $j$-fold composition of the difference operator $\mathcal{D}\left[\left(a_{n}\right)\right]=\left(a_{n}-a_{n-1}\right)$, where we define $a_{n}=0$ for all $n \leq 0$. Let $\left(b_{m, n}\right)=\mathcal{D}^{m}\left[\left(w_{k, n} g(n)\right)\right]$, and let $\widetilde{I}_{k}=\bigcup_{j=k-1}^{k+2} I_{j}, n_{k}=2^{k+2}$ (one more than the largest element of $\left.\widetilde{I}_{k}\right)$. Since $w_{k, n}=w_{k}(n)$, where $w_{k}(x)=\phi\left(x 2^{1-k}\right)$, and $\phi(x)$ is a $C^{\infty}$ function which is constant outside [1,4], it is easy to see that $w_{k}^{(i)} \leq C x^{-i}$ for all $0 \leq i \leq m$, where $C$ depends on $m$, but not on $k$. It follows that for $i \leq m$, the $i$ th derivative of $g(x) w_{k}(x)$ is uniformly (in $k$ ) bounded by $C x^{t+1 / p-1-i} v_{k}$, and so $\left|b_{i, n}\right| \lesssim 2^{(t+1 / p-1-i) k} v_{k}$ when $n \in \widetilde{I}_{k}$. On the other hand, $b_{i, n}=0$ when $n \notin \widetilde{I}_{k}$, since $w_{k, n}=0$ when $n \notin I_{k} \cup I_{k+1}$, and $2^{k-2} \geq m$.

By partial summation we see that

$$
\begin{aligned}
\widetilde{\Delta}_{k} f(z)=\sum_{n=2}^{\infty} w_{k, n} g(n) z^{n} & =\sum_{n \in \widetilde{I}_{k}} b_{1, n} \sum_{n \leq m \in \widetilde{I}_{k}} z^{m} \\
& =\sum_{n \in \widetilde{I}_{k}} b_{1, n} \frac{z^{n}-z^{n_{k}}}{1-z}=\sum_{n \in \widetilde{I}_{k}} b_{1, n} \frac{z^{n}}{1-z}
\end{aligned}
$$

where the last line follows from the fact that $\sum_{n \in \widetilde{I}_{k}} b_{1, n}=0$. Since $\sum_{n \in \widetilde{I}_{k}} b_{i, n}=0$ for all $1 \leq i \leq m$, we can repeat this partial summation argument to get

$$
\widetilde{\Delta}_{k} f(z)=\sum_{n=2}^{\infty} w_{k, n} g(n) z^{n}=\sum_{n \in \widetilde{I}_{k}} b_{m, n} \frac{z^{n}}{(1-z)^{m}} .
$$

Estimating as before, we get that for $|\theta| \in\left[2^{-j}, 2^{-j+1}\right],-1 \leq j \leq k$,

$$
\left|\widetilde{\Delta}_{k} f\left(e^{i \theta}\right)\right|^{p} \leq 2^{(k+2) p} \cdot 2^{k(p t+1-p-m p)} v_{k}^{p} \cdot 2^{j m p} \lesssim 2^{m p(j-k)+k} \cdot 2^{k p t} v_{k}^{p} .
$$

Since $m>1 / p$, we can integrate these estimates to get

$$
I I \lesssim 2^{k p t} v_{k}^{p} \cdot \sum_{j=-1}^{k} 2^{(m p-1)(j-k)} \lesssim 2^{k p t} v_{k}^{p}
$$

We can now characterize $S_{2}(X)$ and $s_{2}(X)$ when $X$ is any $H(p, q, t)$ space.

Theorem 4.2. If $s \in \mathbb{R}, 0<p, q \leq \infty$, then

$$
\begin{aligned}
& s_{2}(H(p, q, t))= \begin{cases}D^{t} l(2, q), & 0<p \leq 2, \\
D^{t} l\left(p^{\prime}, q\right), & 2 \leq p \leq \infty,\end{cases} \\
& S_{2}(H(p, q, t))= \begin{cases}D^{t+1 / p-1} l(\infty, q), & 0<p \leq 1, \\
D^{t} l\left(p^{\prime}, q\right), & 1 \leq p \leq 2, \\
D^{t} l(2, q), & 2 \leq p \leq \infty .\end{cases}
\end{aligned}
$$

At least one direction of each containment in this theorem follows from Lemma 2.5. The proofs for the opposite directions involves detailed case analyses, but the underlying 
idea is rather simple: all necessary counterexamples are lacunary or monotonic. If $p \neq$ $\infty$, membership of each counterexample in $H(p, q, s)$ is determined by one of Lemmas $2.2,2.3,4.1$ (depending on whether the sequence is lacunary, eventually monotonic with $p>1^{1}$, or eventually monotonic with $p \leq 1$ ), while membership in any $F_{2}$ space is determined by direct calculation. The main task for each finite $p$ case is thus to write down a counterexample which works - we shall do this and, with the above hints, we leave the detailed verification of membership and non-membership to the reader in each case, with the occasional exception of the case $p=\infty$ which requires some special arguments. It is convenient for our proof to define the "lacunarizing" operator Lac on the space of all sequences by $\operatorname{Lac}\left[\sum_{n=1}^{\infty} a_{n} x^{n}\right]=\sum_{n=1}^{\infty} a_{n} x^{2^{n}}$.

Proof of Theorem 4.2. In view of the action of fractional differentiation on $H(p, q, t)$, we may as well assume that $t=1$. We first consider $s_{2}(H(p, q, 1))$. The fact that this space contains $D^{1} l\left(\min \left\{p^{\prime}, 2\right\}, q\right)$, and equals it if $p \leq 2$, follows immediately from Lemma 2.5. By combining this with Lemma 2.7, other containments of the form $D^{s} l(u, v) \subset H(p, q, 1)$ follow; we call these the trivial containments. We need to show that, for each choice of $u, v$, no larger values of $s$ are possible. We assume, as we may, that $p>2$.

Suppose first that $u \leq p^{\prime}$. For $v \leq q$, we get the trivial containment $D^{1} l(u, v) \subset$ $H(p, q, 1)$. This is sharp since $\left(a_{n}\right) \equiv \operatorname{Lac}\left[\left(n 2^{n}\right)\right] \in D^{s} l(u, v) \backslash H(p, q, 1)$, for all $s>1$ (if $p=\infty$, we can still use Lemma 2.2 to get that $\left(a_{n}\right)$ does not lie in $H(2, q, 1)$, and hence not in the smaller space $H(\infty, q, 1)$. For $v>q$, we get the trivial containments $D^{s} l(u, v) \subset$ $H(p, q, 1)$ for all $s<1$. This is sharp since $\operatorname{Lac}\left[\left(n^{-1 / q} 2^{n}\right)\right] \in D^{1} l(u, v) \backslash H(p, q, 1)$; the case $p=\infty$ is handled as before.

Suppose instead that $u>p^{\prime}$. If $v \leq q$, we get the trivial containment $D^{s} l(u, v) \subset$ $H(p, q, 1)$, for $s=1 / u+1 / p$. This is sharp for $p<\infty$ since $D^{1 / p+\epsilon / 2} \in D^{s} l(u, v) \backslash$ $H(p, q, 1)$, whenever $s=1 / u+1 / p+\epsilon, \epsilon>0$. For $p=\infty$, we instead note that $D^{\epsilon / 2} \in$ $D^{1 / u+\epsilon} l(u, v) \backslash H(4 / \epsilon, q, 1)$, for every $0<\epsilon<1$. On the other hand, if $v>q$, we get the trivial containment $D^{s} l(u, v) \subset H(p, q, 1)$, for all $s<s_{0} \equiv 1 / u+1 / p$. This is sharp for $p<\infty$ since $D^{1 / p}\left(\log ^{-1 / q}(n+2)\right) \in D^{s_{0}} l(u, v) \backslash H(p, q, 1)$.

Sharpness for $p=\infty$ (and $u>p^{\prime}, v>q$ ) is similar in spirit, but we cannot use Lemma 2.3. We first write $f_{k}(z) \equiv\left(z^{2^{k+1}}-z^{2^{k}}\right) /(z-1)$ for each $k \in \mathbb{N}$. Note that $f_{k}(r) \geq 0$ for all $0<r<1$, and that there exists a constant $c$ independent of $k$ such that $f_{k}(r) \geq 2^{k} c$ for all $r \in\left[1-2^{-k}, 1-2^{-k-1}\right]$. Defining $f=\sum_{k=1}^{\infty} k^{-1 / q} f_{k}$, it is easy to deduce that $f \notin H(\infty, q, 1)$. The Taylor coefficients of $f$ in the dyadic block $I_{k}$ are all $k^{-1 / q}$, and so $f \in D^{1 / u} l(u, v)$.

We must still consider $S_{2}(H(p, q, 1))$. The fact that this space is contained in the indicated $F_{2}$ space, and equals it if $p \geq 2$, follows immediately from Lemma 2.5. By combining this with Lemma 2.7 , we readily deduce other containments of the form $H(p, q, 1) \subset D^{s} l(u, v)$, which we again call the trivial containments. We need to show that no containments with smaller values of $s$ are possible. We assume, as we may, that $p<2$.

\footnotetext{
${ }^{1}$ Because the point-evaluation functionals are bounded on $H(p, q, t)$ spaces, the "if and only if"
} conclusion in Lemma 2.3 is valid for sequences that are merely eventually monotonic. 
Case 1: $1<p<2$

Suppose that $q \leq v$. If $p^{\prime} \leq u$, we get the trivial containment $H(p, q, 1) \subset D^{s} l(u, v)$ for $s=1$, while if $p^{\prime}>u$, we get this containment with $s=1 / u+1 / p$. We see that $s$ is minimal for $p^{\prime} \leq u$ by Lemma 2.6, and for $p^{\prime}>u$ because $D^{1 / p-\epsilon / 2} \in H(p, q, 1) \backslash D^{s-\epsilon} l(u, v)$, for all $\epsilon>0$. We may therefore assume that $q>v$.

If $p^{\prime} \leq u$, we trivially get $H(p, q, 1) \subset D^{s} l(u, v)$ for all $s>s_{0}=1$, while if $p^{\prime}>u$, we trivially get $H(p, q, 1) \subset D^{s} l(u, v)$ for all $s>s_{0}=1 / u+1 / p$. We cannot take $s=s_{0}$ when $p^{\prime} \leq u$, because $\operatorname{Lac}\left[\left(n^{-1 / v} 2^{n}\right)\right] \in H(p, q, 1) \backslash D^{1} l(u, v)$, nor when $p^{\prime}>u$, since $D^{1 / p}\left(\log ^{-1 / v}(n+2)\right) \in H(p, q, 1) \backslash D^{s_{0}} l(u, v)$.

Case 2: $p \leq 1$

Here we only need to consider two cases for a containing space $D^{s} l(u, v)$. If $q \leq v$, containment is trivially true for $s=1 / u+1 / p$. If $s=1 / u+1 / p-\epsilon, \epsilon>0$, then $D^{1 / p-\epsilon / 2} \in$ $H(p, q, 1) \backslash D^{s} l(u, v)$ (or we may avoid using Lemma 4.1 by instead using the function $f(z)=(1-z)^{-1-1 / p+\epsilon / 2}$ as in Lemma 2.6). On the other hand, if $q>v$, containment is trivially true for all $s>1 / u+1 / p$. To prove minimality, note that $D^{1 / p}\left(\log ^{-1 / v}(n+2)\right)$ lies in $H(p, q, 1) \backslash D^{1 / u+1 / p} l(u, v)$.

Note that if $F \subset F^{\prime}$ are families of sequence spaces, and $X$ is a sequence space, then $s_{F}(X)=s_{F}\left(s_{F^{\prime}}(X)\right)$ and $S_{F}(X)=S_{F}\left(S_{F^{\prime}}(X)\right)$. Using these identities in the case $F_{1} \subset F_{2}$, together with Theorem 4.2 with Lemma 3.1, we get an explicit description of $s_{1}(H(p, q, t))$ and $S_{1}(H(p, q, t))$. Since in most cases there is another bifurcation between large and small $q$, we record the answer only for $q=p$. Even with this restriction, it becomes clear that $F_{2}$ is more natural than $F_{1}$ for helping to describe $H(p, q, t)$.

Corollary 4.3. For all $t \in \mathbb{R}$,

$$
\begin{aligned}
& s_{1}(H(p, p, t))=\left\{\begin{array}{lr}
D^{t}\left[l^{p}+\bigcup_{\epsilon>0} D^{-\epsilon} l^{2}\right], & 0<p<2, \\
D^{t}\left[l^{p^{\prime}}+D^{2 / p-1} l^{p}\right], & 2 \leq p \leq \infty,
\end{array}\right. \\
& S_{1}(H(p, p, t))=\left\{\begin{array}{lr}
D^{t}\left[D^{1 / p-1} l^{\infty} \cap D^{2 / p-1} l^{p}\right], & 0<p \leq 1, \\
D^{t}\left[l^{p^{\prime}} \cap D^{2 / p-1} l^{p}\right], & 1 \leq p \leq 2, \\
D^{t}\left[l^{p} \cap\left(\bigcap_{\epsilon>0} D^{\epsilon} l^{2}\right)\right], & 2<p \leq \infty .
\end{array}\right.
\end{aligned}
$$

\section{Applications to multipliers}

We shall calculate $s_{2}$, and often also $S_{2}$, for the multiplier spaces $X \equiv(H(p, q, t), l(u, v))$ and $Y \equiv(l(u, v), H(p, q, t))$. Our results allow us to determine exactly when these spaces have the form $D^{s} l(a, b)$, and we also determine the values of $s, a, b$ whenever $X$ or $Y$ have this form. Some results of this type are to be found in the literature, notably the papers of Blasco [3] and of Jevtić and Pavlović [11]. Assuming that $u=v$, Blasco characterizes $X$ when $p \geq 2$, when $p, q \leq 1 \leq u$, and when $p \leq 1 \leq q \leq u$ (Theorems 4.1,4.2, 4.3 $3^{2}$ of [3]). 
Jevtić and Pavlović characterize $X$ in all $u=v$ cases except for $1<p<2, u<p^{\prime}$. The answer in all of these cases is of the form $D^{s} l(a, b)$, and we recover these results below. Jevtić and Pavlović conjecture that the answer for the missing $u=v$ case is a specific space of the form $D^{s} l(a, b)$. We do not find $X$ in the missing case, but we prove that this conjecture is always false. Our results cover all cases (including infinite exponents) and follow rather easily (certainly, in the case of the $s_{2}$-type results). We do of course use Theorem 4.2, whose proof required some effort, but at least the methods used in its proof were consistent in all cases. Thus Theorem 4.2 has a unifying role, replacing a variety of arguments used to prove various special cases in the literature.

Our first theorem examines $(l(u, v), H(p, q, t))$, and the second $(H(p, q, t), l(u, v))$. The cases $p \leq 2$ in the first, and $p \geq 2$ in the second are included for completeness, but are very easy; for instance, since $l(u, v)$ is solid, Lemmas 2.5 and 2.8 imply that for $p \leq 2$,

$$
(l(u, v), H(p, q, t))=\left(l(u, v), s(H(p, q, t))=\left(l(u, v), D^{t} l(2, q)\right)=D^{t} l(2 \ominus u, q \ominus v) .\right.
$$

Theorem 5.1. For arbitrary $t \in \mathbb{R}, 0<p, q, u, v \leq \infty, s_{2}(l(u, v), H(p, q, t))$ and $S_{2}(l(u, v), H(p, q, t))$ are given by the following table.

\begin{tabular}{|c|c|c|}
\hline & $s_{2}(l(u, v), H(p, q, t))$ & $S_{2}(l(u, v), H(p, q, t))$ \\
\hline $0<p \leq 2$ & $D^{t} l(2 \ominus u, q \ominus v)$ & $D^{t} l(2 \ominus u, q \ominus v)$ \\
\hline $2<p<\infty$ & $D^{t} l\left(p^{\prime} \ominus u, q \ominus v\right)$ & $U$ \\
\hline$p=\infty$ & $D^{t} l(1 \ominus u, q \ominus v)$ & $D^{t} l(1 \ominus u, q \ominus v)$ \\
\hline
\end{tabular}

The missing entry $U$ satisfies $D^{t} l\left(p^{\prime} \ominus u, 2 \ominus u, q \ominus v\right) \subset U \subset D^{t} l(2 \ominus u, q \ominus v)$.

Proof. By Lemma 3.2, $s_{2}(l(u, v), H(p, q, t))=\left(l(u, v), s_{2}(H(p, q, t))\right)$. Thus calculating $s_{2}(l(u, v), H(p, q, t))$ can be done in all cases by means of Theorem 4.2 and Lemma 2.8 . Similarly, $S_{2}(l(u, v), H(p, q, t))$ is contained in $D^{t} l(2 \ominus u, q \ominus v)$ if $2<p<\infty$. Since the case $p \leq 2$ is easy, it is left to prove that

$$
\begin{aligned}
& S_{2}(l(u, v), H(p, q, t)) \supset D^{t} l\left(p^{\prime} \ominus u, 2 \ominus u, q \ominus v\right), \quad 2<p<\infty, \\
& S_{2}(l(u, v), H(p, q, t)) \subset D^{t} l(1 \ominus u, q \ominus v), \quad p=\infty .
\end{aligned}
$$

By Lemma 2.4, $l\left(p^{\prime}, 2\right) \subset H^{p}$, and so $l\left(p^{\prime} \ominus u, 2 \ominus u\right) \subset\left(l^{u}, H^{p}\right)$. Using (2.2) and Lemma 2.9, (5.1) follows immediately.

Containment (5.2) is equivalent to $(l(u, v), H(\infty, q, t)) \subset D^{t} l(1 \ominus u, q \ominus v)$, so we prove the latter. We may assume without loss of generality that $t=1$. Suppose for the purposes of contradiction that the required result is false. Since $l(u, v)$ is solid, $(l(u, v), H(\infty, q, t))$ is also solid, and so we can find a non-negative sequence $\lambda \in(l(u, v), H(\infty, q, 1)) \backslash D^{1} l(1 \ominus$ $u, q \ominus v)$. Since $D^{1} l(1 \ominus u, q \ominus v)=\left(l(u, v), D^{1} l(1, q)\right)$, we can find a non-negative sequence

${ }^{2}$ As stated, this theorem is inconsistent with our results; the parameter $r$ should be simply $s$. The error in the proof arises where $1 / t$ is defined to be $1-q / s$, rather than the correct $1 / q-1 / s$. 
$a \in l(u, v)$ such that $\lambda a \notin D^{1} l(1, q)$. By hypothesis, $f(z) \equiv \sum_{n=1}^{\infty} \lambda_{n} a_{n} z^{n} \in H(\infty, q, 1)$. However, for each $k \geq 1$,

$$
M_{\infty}(r, f) \geq f(r) \geq\left(\Delta_{k} f\right)(r) \gtrsim\left\|S_{k}(\lambda a)\right\|_{l^{1}}, \quad r \in\left[1-2^{-k}, 1-2^{-k-1}\right] .
$$

Since $\left(2^{-k}\left\|S_{k}(\lambda a)\right\|_{l^{1}}\right) \notin l^{q}$, it follows by routine estimation that $f \notin H(\infty, q, 1)$, giving the required contradiction.

Theorem 5.2. For arbitrary $t \in \mathbb{R}, 0<p, q, u, v \leq \infty, s_{2}(H(p, q, t), l(u, v))$ and $S_{2}(H(p, q, t), l(u, v))$ are given by the following table.

\begin{tabular}{|c|c|c|}
\hline & $s_{2}(H(p, q, t), l(u, v))$ & $S_{2}(H(p, q, t), l(u, v))$ \\
\hline $0<p \leq 1$ & $D^{-t-1 / p+1} l(u, v \ominus q)$ & $D^{-t-1 / p+1} l(u, v \ominus q)$ \\
\hline $1<p<2$ & $D^{-t} l\left(u \ominus p^{\prime}, v \ominus q\right)$ & $V$ \\
\hline $2 \leq p \leq \infty$ & $D^{-t} l(u \ominus 2, v \ominus q)$ & $D^{-t} l(u \ominus 2, v \ominus q)$ \\
\hline
\end{tabular}

The missing entry $V$ satisfies $D^{-t} l\left(u \ominus p^{\prime}, u \ominus 2, v \ominus q\right) \subset V \subset D^{-t} l(u \ominus 2, v \ominus q)$.

Proof. Most of the proof is easy, and similar to that of Theorem 5.1. The one part that is genuinely different is the fact that $S_{2}(H(p, q, t), l(u, v))$ is a subset of $D^{-t-1 / p+1} l(u, v \ominus q)$, when $0<p \leq 1$. Here, we may assume that $t=1$, in which case this containment is equivalent to $(H(p, q, 1), l(u, v)) \subset D^{-1 / p} l(u, v \ominus q)$. For the purposes of contradiction, we assume that this is false. By solidity, we can find a non-negative sequence $\lambda \in(H(p, q, 1), l(u, v)) \backslash D^{-1 / p} l(u, v \ominus q)$. Since $D^{-1 / p} l(u, v \ominus q)=\left(D^{1 / p} l(\infty, q), l(u, v)\right)$, there exists $a \in D^{1 / p} l(\infty, q)$ such that $\lambda a \notin l(u, v)$. We may assume that $a_{n} \geq 0$ and that $a_{n}$ has a constant value $b_{k}$ in each block $I_{k}$ (if necessary, replace $a_{n}$ by $\left\|S_{k} a\right\|_{l^{\infty}}$ for each $n \in I_{k}$ ). Choosing a non-negative bump function $\phi$ which equals 1 on $[1,2]$, and is supported on [3/4,3], we define $f(z)=\sum_{n=1}^{\infty} g(n) z^{n}$, where $g(x)=\sum_{k=1}^{\infty} b_{k} \phi\left(2^{1-k} x\right)$. It is routine to check that $g:[1, \infty) \rightarrow \mathbb{R}$ is a $C^{\infty}$ function satisfying $g^{(i)}(x) \leq C_{m} x^{1 / p-i} u_{k}$, for all $2^{k-1} \leq x<2^{k}, 0 \leq i \leq m$, where $u_{k}=2^{-k / p}\left(b_{k-1}+b_{k}+b_{k+1}\right)$ and $\left(u_{k}\right) \in l^{q}$. It follows from Lemma 4.1 that $f \in H(p, q, 1)$ and so $\left(\lambda_{n} \hat{f}(n)\right) \in l(u, v)$. By construction, $\hat{f}(n) \geq a_{n}$, leading to a contradiction since $\lambda a \notin l(u, v)$.

Remarks.

(1) These theorems characterize multipliers between all $l(u, v)$ spaces and the Bloch space $\mathcal{B}=H(\infty, \infty, 0)$ :

$$
\begin{aligned}
(l(u, v), \mathcal{B}) & =l(1 \ominus u, \infty), \\
(\mathcal{B}, l(u, v)) & =l(u \ominus 2, v) .
\end{aligned}
$$

(2) Note that for any family $F$ and spaces $A, B,(A, B)=s_{F}(A, B)$ if $s_{F}(A, B)=$ $S_{F}(A, B)$, whereas $(A, B)$ cannot be a member of $F$ if $s_{F}(A, B) \neq S_{F}(A, B)$. Thus, Theorem 5.1 gives $(l(u, v), H(p, q, t))$ in all cases except $2<p<\infty, u>p^{\prime}$ (the spaces containing, and contained in, $U$ are equal if $\left.u \leq p^{\prime} \leq 2\right)$. In the missing case, 
$X \equiv(l(u, v), H(p, q, t))$ cannot have the form $D^{s} l(b, c)$ since $p^{\prime} \ominus u<2 \ominus u$, and so $s_{2}(X)=D^{t} l\left(p^{\prime} \ominus u, q \ominus v\right)$ is a proper subset of $D^{t} l\left(p^{\prime} \ominus u, 2 \ominus u, q \ominus v\right) \subset S_{2}(X)$.

(3) Arguing as in $(2),(H(p, q, t), l(u, v))$ is given by Theorem 5.2 in all cases except $1<p<2, u<p^{\prime}$, and in this missing case it cannot have the form $D^{s} l(b, c)$. This disproves a conjecture in [11] that it equals $D^{-t} l\left(u \ominus p^{\prime}, u \ominus q\right)$ if $u=v$.

(4) The proofs in this section can be modified to derive other containments not mentioned above. For instance, using Lemma 2.4, we see that for $1<p<2,\left(H^{p}, l^{u}\right)$ contains $l\left(u \ominus p^{\prime}, u \ominus 2, \ldots, u \ominus 2\right)$, where the parameter " $u \ominus 2$ " may be repeated any finite number of times. Consequently, the missing space $V \equiv D^{-t} l^{v \ominus q}\left(H^{p}, l^{u}\right)$ contains $D^{-t} l\left(u \ominus p^{\prime}, u \ominus 2, \ldots, u \ominus 2, v \ominus q\right)$. However, according to Theorem 5.2, it does not contain $D^{-t} l(u \ominus 2, v \ominus q)$ if $u<p^{\prime}$. This suggests that $V$ may be difficult to describe in the missing case; a similar analysis applies to the missing case of Theorem 5.1.

Note also that $\left(l^{\infty}, H(p, q, t)\right)=s(H(p, q, t))$, a space which appears to be unknown when $2<p<\infty$, and so it is not surprising that $(l(u, v), H(p, q, t))$ appears to be difficult to determine for large $u$. An easy duality argument implies that $(H(p, q, t), l(u, v))=\left(l\left(u^{\prime}, v^{\prime}\right), H\left(p^{\prime}, q^{\prime},-t\right)\right)$ as long as $p, q, u, v \in[1, \infty)$, so it is similarly not surprising that $(H(p, q, t), l(u, v))$ is difficult to determine when $1<p<2$ and $u$ is small.

(5) It is perhaps appropriate to repeat our warning in Section 1 that $H(p, \infty, 0)$, as we have defined it, is not the same as $H^{p}$. Thus, for instance, Theorem 5.2 says that $\left(H(1, \infty, 0), l^{1}\right)=l^{1}$, but it is certainly not true that $\left(H^{1}, l^{1}\right)=l^{1}$, since lacunary $H^{1}$ functions coincide with lacunary $l^{2}$, and so $\left(H^{1}, l^{1}\right)$ must contain at least the lacunary elements of $l^{2}$ by Hölder's inequality. A characterization of $\left(H^{1}, l^{1}\right)$, due to C. Fefferman, is to be found at the end of [1].

(6) One cannot change containment to equality in parts (iii) and (iv) of Lemma 3.2: for (iii), consider the case $p \leq 1$ of Theorem 5.2, and for (iv), consider the case $p=\infty, u>1$ of Theorem 5.1.

\section{REFERENCES}

[1] J.M. Anderson and A.L. Shields, Coefficient multipliers on Bloch functions, Transactions of the American Mathematical Society 224 (1976), 255-65.

[2] G. Bennett, D.A. Stegenga, and R. Timoney, Coefficients of Bloch and Lipschitz functions, Illinois Journal of Mathematics 25 (1981), 520-31.

[3] O. Blasco, Multipliers on spaces of analytic functions, Canadian Journal of Mathematics 47 (1995), 44-64.

[4] S. Buckley, Mixed norms and analytic function spaces, Proceedings of the Royal Irish Academy 100A (2000), 1-9.

[5] S. Buckley, P. Koskela, and D. Vukotić, Fractional integration, differentiation, and weighted Bergman spaces, Mathematical Proceedings of the Cambridge Philosophical Society 126 (1999), 369-85.

[6] S. Buckley, M.S. Ramanujan, and D. Vukotić, Bounded and compact multipliers between Bergman and Hardy spaces, Integral Equations and Operator Theory 35 (1999), 1-19.

[7] P.L. Duren, Theory of $H^{p}$ spaces, Academic Press, New York, 1970.

[8] T. Flett, The dual of an inequality of Hardy and Littlewood and some related inequalities, Journal of Mathematical Analysis and Applications 38 (1972), 746-65. 
[9] D.J.H. Garling, On topological sequence spaces, Mathematical Proceedings of the Cambridge Philosophical Society 63 (1967), 997-1019.

[10] C.A. Horowitz, Zeros of functions in the Bergman spaces, Duke Journal of Mathematics 41 (1974), 693-710.

[11] M. Jevtić and M. Pavlović, Coefficient multipliers on spaces of analytic functions, Acta Universitatis Szegediensis. Acta Scientiarum Mathematicarum 64 (1998), 531-45.

[12] C.N. Kellogg, An extension of the Hausdorff-Young Theorem, Michigan Mathematics Journal 18 (1971), 121-7.

[13] J.E. Littlewood, Mathematical notes (13): On mean values of power series (II), Journal of the London Mathematical Society 5 (1930), 179-82.

[14] M. Mateljević and M. Pavlović, $L^{p}$ behaviour of the integral means of analytic functions, Studia Mathematica 77 (1984), 219-37.

[15] A. Nakamura, F. Ohya, and H. Watanabe, On some properties of functions in weighted Bergman spaces, Proceedings of the Faculty of Science of Tokai University 15 (1979), 33-44.

[16] A. Pietsch, Verallgemeinerte vollkommene Folgenräume, Studia Mathematics (Ser. Specjalna) Zeszyt 1 (1963), 89-91.

[17] M.S. Ramanujan and N. Tanović-Miller, A generalization of the Hausdorff-Young Theorem, Acta Mathematica Hungarica 81 (1998), 279-305. 